\title{
The AMBRE Project: [Y/Mg] stellar dating calibration with Gaia
}

\author{
A. Titarenko ${ }^{1}$, A. Recio-Blanco ${ }^{1}$, P. de Laverny ${ }^{1}$, M. Hayden ${ }^{1,2,3}$, and G. Guiglion ${ }^{4,1}$ \\ ${ }^{1}$ Laboratoire Lagrange (UMR7293), Université de Nice Sophia Antipolis, CNRS, Observatoire de la Côte d'Azur, BP 4229, 06304 \\ Nice Cedex 4, France \\ e-mail: 2chlaidze@gmail.com \\ 2 Sydney Institute for Astronomy (SIfA), School of Physics, A28, The University of Sydney, NSW 2006, Australia \\ ${ }^{3}$ Center of Excellence for Astrophysics in Three Dimensions (ASTRO-3D), Australia \\ ${ }^{4}$ Leibniz-Institut für Astrophysik Potsdam (AIP), 14482 Potsdam, Germany
}

Received 26 June 2018 / Accepted 8 November 2018

\begin{abstract}
Chemical abundance dating methods open new paths for temporal evolution studies of the Milky Way stellar populations. In this paper, we use a high spectral resolution database of turn-off stars in the solar neighbourhood to study the age dependence of the [Y/Mg] chemical abundance ratio. Our analysis reveals a clear correlation between $[\mathrm{Y} / \mathrm{Mg}]$ and age for thin disc stars of different metallicities, in synergy with previous studies of solar-type stars. In addition, no metallicity dependence with stellar age is detected, allowing us to use the $[\mathrm{Y} / \mathrm{Mg}]$ ratio as a reliable age proxy. Finally, the $[\mathrm{Y} / \mathrm{Mg}]-$ age relation presents a discontinuity between thin and thick disc stars around 9-10 Gyr. For thick disc stars, the correlation has a different zero point and probably a steeper trend with age, reflecting the different chemical evolution histories of the two disc components.
\end{abstract}

Key words. Galaxy: abundances - Galaxy: disk - Galaxy: stellar content - Galaxy: structure

\section{Introduction}

Since the advent of stellar astrophysics, the estimation of stellar ages has been a crucial challenge with many fundamental implications. Starting with the historical debate around the age of our Sun, stellar ages have been recognised as clocks of the evolving Universe that we wish to understand. They are the basis of stellar evolution studies, of the stellar properties coded in chemical evolution analysis, and more generally a key piece of information of our understanding of any stellar population.

Numerous dating methods have been developed based on different physical processes and models. Nuclear cosmochronology (Hoyle \& Fowler 1960; Bland-Hawthorn \& Freeman 2014), isochrone fitting (first discovered by Schaltenbrand 1974; Luri et al. 1992; Valls-Gabaud 2014), asteroseismology techniques (Pesnell 1987; Guzik et al. 2000), and more indirect chemical proxies of stellar age have been used and applied to very different samples of stars. In particular, for Galactic Archaeology studies, dating techniques applicable to large numbers of objects are crucial. In this sense, Bayesian isochrone fitting methods (Jørgensen \& Lindegren 2005), based on the comparison of the absolute luminosity (or magnitude) of stars and their effective temperatures (or colour) with theoretical models and through Bayesian methods, are among the most commonly used. However, one of the main weaknesses of this approach for Galactic archaeology studies is that it can give reliable results only for stars at the turn-off and the subgiant branch stages, as far as the isochrones are more age sensitive for this type of stars. In addition, precise age estimation from isochrone fitting depends strongly on our knowledge of the stellar distance.

Before the advent of the European Space Agency Gaia mission (Gaia Collaboration 2016b), precise stellar distances were constrained to the solar neighbourhood. The current, unprecedented precision of the Gaia astrometric measurements allows us to perform the direct distance estimation for about 1.3 billion stars. This opens the way to isochrone fitting age estimations for large samples of stars. On the one hand, Gaia ages are already revolutionising our view of the Milky Way stellar populations (Hayden et al. 2018). On the other hand, they will permit the calibration of secondary clocks, like chemical abundance age proxies. These more indirect techniques are important to extend the possibility of stellar dating outside the sphere of the Gaia precise distances and to stars in different stellar evolutionary stages.

This paper focuses on a particular chemical clock: the yttrium to magnesium abundance ratio. Since the studies of da Silva et al. (2012), Nissen (2015), Spina et al. (2016), Tucci Maia et al. (2016), and the recent work of Anders et al. (2018), a correlation of [Y/Mg] with stellar age for solar twins has been noticed. In the work of Feltzing et al. (2017) a strong correlation is found only for stars with $[\mathrm{Fe} / \mathrm{H}]>-0.5 \mathrm{dex}$, and Slumstrup et al. (2017) find that this relation also works for solar metallicity stars over the helium-core-burning phase.

Finally, it should be noted that chemical dating can be closely linked to the chemical evolution of a stellar population. In this sense, the comparison of results between thin and thick disc stars can shed a light on the different evolutionary paths of these two disc components. In this paper, a chemical definition of the thin/thick disc populations has been followed. As revealed by several studies inside and outside the solar neighbourhood, thick and thin disc stars seem to separate in two sequences in the $[\alpha / \mathrm{Fe}]$ versus metallicity plane (e.g. Adibekyan et al. 2012; Recio-Blanco et al. 2014). Thick disc stars have higher $[\alpha / \mathrm{Fe}]$ abundances than thin disc stars of the same metallicity, showing also a faster evolution of their $[\alpha / \mathrm{Fe}]$ abundance with time (Haywood et al. 2013; Hayden et al. 2018). 
The aim of our work is to analyse the age dependence of the [Y/Mg] ratio for turn-off stars in a wide range of metallicities, using a homogeneous and high-resolution spectroscopic sample from the AMBRE project (de Laverny et al. 2012, 2013). To this purpose, isochrone fitting ages using astrometric Gaia data from the first data release (Gaia Collaboration 2016a) are used. The high precision of chemical data and of stellar ages, combined with the important number of analysed stars (342 turn-off stars in a wide range of metallicities instead of less than 100, mostly solar twins, for the majority of the previous studies), helps us to further explore the nature and precision of the $[\mathrm{Y} / \mathrm{Mg}]$ ratio as an age indicator.

In the following section we describe the data set used in this work. Sections 3 and 4 present the methodology used for the chemical abundance estimation and the corresponding error analysis. The main results are described in Sects. 5 and 6, while Sect. 7 discusses the differences encountered between the thin and the thick disc populations. The final conclusions are summarised in Sect. 8.

\section{The AMBRE-TGAS data}

The Archeologie avec Matisse Basée sur les aRchives de l'ESO (AMBRE) project, established by ESO and l'Observatoire de la Côte d'Azur in 2009 (de Laverny et al. 2013), concerns the parameterisation of the stellar high-resolution spectra of the ESO archives. Stellar spectra collected by three high-resolution spectrographs have already been analysed (FEROS, HARPS, UVES). In this paper, we concentrate on the 4355 parametrised HARPS spectra for which the best stellar $T_{\text {eff }}, \log (g),[\mathrm{Fe} / \mathrm{H}]$, and radial velocity are available (De Pascale et al. 2014). Within the AMBRE database, the HARPS spectra have the highest spectral resolution and contain an important number of stars at the turnoff stage for which isochrone ages can be reliably derived.

The robust identification of the AMBRE/HARPS targets was carefully addressed to overcome the challenges of a catalogue that is build up from archival data. The 2MASS catalogue was chosen as a reference because it offered the advantage of providing photometric information for the targets. The crossmatch between the AMBRE/HARPS and 2MASS coordinates was performed using the xcross program (ESO client). In the case of multiple matches within the radius of search $(5 \operatorname{arcsec}$ for AMBRE/HARPS), a comparison of the spectroscopic and the photometric effective temperatures was performed. For each match from the 2MASS catalogue, the photometric effective temperature was calculated using the relations of Alonso et al. (1996) and constants from González Hernández \& Bonifacio (2009). When there were multiple matches, the objects with the minimal values of $\left|T_{\text {phot }}-T_{\text {AMBRE }}\right|$ were selected. A total of 2416 different stars along the Hertzprung-Russell diagram were found.

The identified stars were then searched in the Tycho-Gaia Astrometric Solution (TGAS) catalogue. Ages were determined using Bayesian analysis by comparing derived stellar parameters from AMBRE/HARPS data $\left(T_{\text {eff }},[\mathrm{Fe} / \mathrm{H}],[\mathrm{Mg} / \mathrm{Fe}]\right.$, $M_{\mathrm{K}}$ ) with theoretical isochrones from the Dartmouth group ${ }^{1}$ (Dotter et al. 2008), using a method similar to that described by Jørgensen \& Lindegren (2005). A complete description of the methods employed in this paper to determine ages are provided by the AMBRE-Gaia DR1 catalogue (Hayden et al. 2018). The isochrones are spaced in age from 0 to $15 \mathrm{Gyr}$, with steps of $100 \mathrm{Myr}$, in $[\mathrm{Fe} / \mathrm{H}]$ from -2.0 to $+0.6 \mathrm{dex}$ in steps of $0.1 \mathrm{dex}$,

\footnotetext{
1 http://stellar.dartmouth.edu/models/isolf_new.html
}

and from 0.0 to $+0.4 \mathrm{dex}$ in $[\mathrm{Mg} / \mathrm{Fe}]$ in steps of $0.2 \mathrm{dex}$. The median variance of the age probability distribution function is about $1.2 \mathrm{Gyr}$ for stars younger than $4 \mathrm{Gyr}$ and $1.8 \mathrm{Gyr}$ for stars between 7 and 10 Gyr. After the cross-match with TGAS, a total of 2087 stars remain, from which 502 are turn-off stars.

The absolute magnitude is derived using a Bayesian approach with the parallax information from Gaia DR1 (Perryman et al. 2014), as described in Hayden et al. (2018). The assumptions adopted for the distance determination are a single exponential disc with a scale length of $2.7 \mathrm{kpc}$ and a scale height of $300 \mathrm{pc}$, and the luminosity function of the solar neighbourhood derived by Robin et al. (2012). To determine the ages, we adopt several simple assumptions. We assume a flat or uniform star formation history, with age spacing provided by the isochrones. The isochrone points are weighted by mass using a Chabrier IMF (Chabrier 2003). We do not assign any assumption based on the metallicity or $\alpha$-element abundances of the star.

\section{Chemical abundance estimation}

The abundances of magnesium and yttrium were homogeneously derived for the complete HARPS-TGAS catalogue. For this purpose, the GAUGUIN method was used (Bijaoui et al. 2012; Guiglion et al. 2016). It is a classical local optimisation method implementing a Gauss-Newton algorithm, based on a local linearisation around a given set of parameters that are associated with a reference synthetic spectrum (via linear interpolation of the derivatives). A few iterations are carried out through linearisation around the new solutions, until the algorithm converges towards the minimum distance between the observed spectrum and the synthetic spectrum library. In practice, we used GAUGUIN together with the stellar parameters independently determined by the algorithm MATISSE (Recio-Blanco et al. 2006; De Pascale et al. 2014) for the AMBRE project. The GAUGUIN code includes a local normalisation step around the selected spectral lines. It is worth noting that GAUGUIN is part of the GSPspec module of the APSIS pipeline (Bailer-Jones et al. 2013) implemented within the Gaia Data Processing and Analysis Consortium. In particular, GSPspec is in charge of deriving parameters and abundances from Gaia RVS spectra (Recio-Blanco et al. 2016).

The reference theoretical spectra grid used by GAUGUIN for the magnesium abundance estimation was the GES synthetic grid of non-rotating FGKM-type spectra, provided by the GES consortium. It is a high-resolution optical synthetic spectra grid in the 4200-6900 $\AA$ wavelength range calculated from the MARCS stellar atmosphere models (Gustafsson et al. 2008) and the GES/Linelist_V5 atomic and molecular line-lists, provided by GES/LineList Group. The microturbulence velocity $(\xi)$ was included in the grid computation by adopting $\xi$ varying as a function of $T_{\text {eff }}, \log (g)$, and [Fe/H], as adopted in the Gaia-ESO Survey (Bergemann et al., in prep., based on $\xi$ determinations from literature samples). The $[\mathrm{Mg} / \mathrm{Fe}]$ variations used for the abundance estimation correspond to the $[\alpha / \mathrm{Fe}]$ variation of the grid, i.e. \pm 0.4 dex around the nominal value with a step of $0.2 \mathrm{dex}$. To be in agreement with the prepared observational HARPS spectra data set, we reduced the resolution of the theoretical spectra to $\sim 110000$.

Regarding the Y abundance estimations, a specific grid of synthetic spectra has also been computed around the YII lines. We globally followed a procedure similar to the one adopted for the AMBRE grid (de Laverny et al. 2012) except that we also considered an enhancement in yttrium varying over a range 


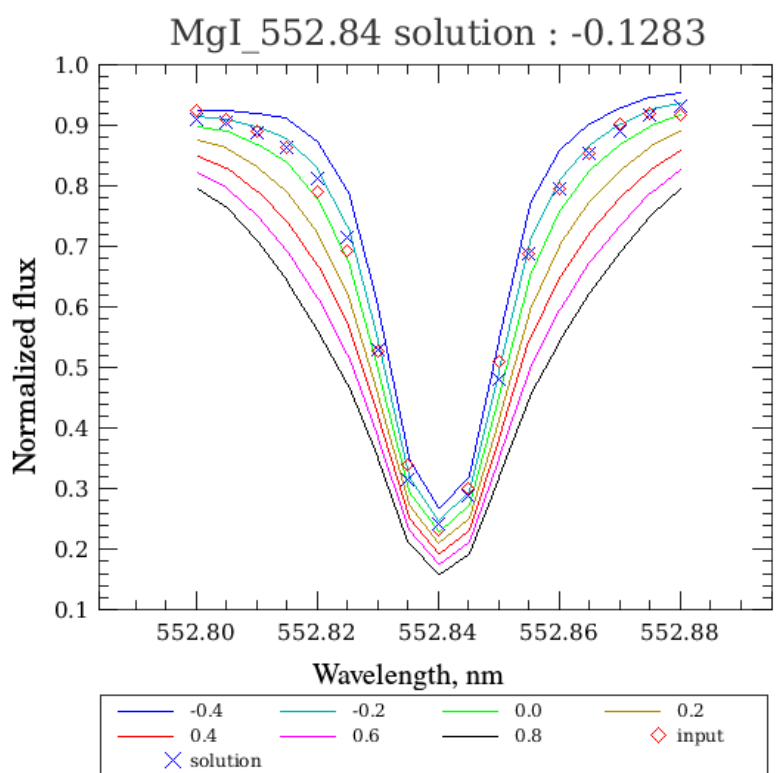

Fig. 1. Example of analysis with the GAUGUIN method. The observed spectrum of HD 9782 is indicated by red circles $\left(T_{\text {eff }}=5980 \mathrm{~K}\right.$, $\log (\mathrm{g})=4.26,[\mathrm{Fe} / \mathrm{H}]=0.08,[\alpha / \mathrm{Fe}]=-0.01)$. The solution is shown with blue crosses.

Table 1. Adopted MgI and YII lines, including the ranges used for abundance estimation with GAUGUIN.

\begin{tabular}{ccc}
\hline \hline MgI line $(\AA)$ & \multicolumn{2}{c}{ Line range $(\AA)$} \\
\hline 5167.3 & 5166.9 & 5167.7 \\
5172.6 & 5172.2 & 5173.15 \\
5183.6 & 5183.2 & 5184.0 \\
5528.4 & 5528.0 & 5528.8 \\
5711.0 & 5710.7 & 5711.4 \\
\hline YII line $(\AA)$ & \multicolumn{2}{c}{ Line range $(\AA)$} \\
\hline 4124.19 & 4124.81 & 4125.01 \\
4398.01 & 4397.86 & 4398.16 \\
4883.68 & 4883.48 & 4883.88 \\
4900.12 & 4899.97 & 4900.27 \\
4982.13 & 4981.98 & 4982.28 \\
5087.42 & 5087.27 & 5087.57 \\
5200.41 & 5200.26 & 5200.56 \\
5728.89 & 5728.69 & 5729.09 \\
\hline
\end{tabular}

of \pm 1.2 dex around the metallicity with a step of $+0.2 \mathrm{dex}$ (leading to 13 different values of $[\mathrm{Y} / \mathrm{Fe}]$ ). We refer to Guiglion et al. (2018) for more details on the computation of this new grid in which we adopted the yttrium line data from Hannaford et al. (1982) and McWilliam et al. (2013; see Table 1).

Our selection of $\mathrm{Mg}$ spectral lines is based on the work of Mikolaitis et al. (2017). We finally retained the five strong lines presented in Table 1 for which the $\mathrm{Mg}$ abundance results were less dispersed. In addition, the selection of Y spectral lines was performed to avoid possible blends (Van der Swaelmen, priv. comm.). Table 2 shows the final line selection, together with the areas for the local spectrum normalization.

The abundances of $\mathrm{Y}$ and $\mathrm{Mg}$ from each spectrum were calculated as the median value of all available the lines (see Table 1), and the result was flagged with a number of calculated lines. As the first quality cut, we adopted the results for the
Table 2. Detail of the number of analysed stars, the single spectrum cases, and the overlap with reference catalogues for the $\mathrm{Y}$ and $\mathrm{Mg}$ abundances.

\begin{tabular}{ccl}
\hline \hline$N_{\text {stars YII }}$ & $N_{\text {stars MgI }}$ & Description \\
\hline 2050 & 1795 & All calculated abundances \\
- & 1670 & In common with Mikolaitis et al. (2017) \\
935 & - & In common with Delgado Mena et al. (2017) \\
\hline 2416 & Stars identified with 2MASS \\
2087 & Subsample containing TGAS stars \\
502 & TO-stars from this TGAS subsample \\
342 & TO-stars with Y and Mg abundances. \\
\hline
\end{tabular}

spectra with $S / N>30$ and standard deviation of median value of the abundance standard deviation smaller than 0.4 dex. The final stellar abundance was then defined as the median abundance value from the multiple spectra of the star. Figure 1 illustrates the process of the abundance estimation with GAUGUIN, for a $\mathrm{Mg}$ line of the HD 9782 star spectrum (open circles). The calculated interpolated synthetic spectra grid for $7 \mathrm{Mg}$ abundance values is colour-coded. The final derived magnesium abundance is shown with $\mathrm{X}$-symbols.

\section{Abundance error estimation}

We performed the abundance error analysis following three different indicators. First, the internal error, given by the line-to-line dispersion per spectra, was considered. The corresponding histograms for the $\mathrm{Mg}$ and $\mathrm{Y}$ abundances are shown in Fig. 2. The peak of the distribution is at 0.07 dex for the $\mathrm{Mg}$ abundance and 0.12 dex for the $\mathrm{Y}$ abundance.

Second, thanks to the significant number of repeats in the catalogue $(40 \%$ of the stars have one spectrum, and $95 \%$ of the stars have been observed less then 30 times; see De Pascale et al. 2014), we were able to evaluate the influence of the parameter uncertainties in the abundance estimation by looking at the spectrum-to-spectrum dispersion. We recall that the AMBRE project provides atmospheric parameters for each individual spectrum of a particular star. The variations in the atmospheric parameters for the repeated spectra with $\mathrm{S} / \mathrm{N}$ higher than 30 are lower than $40 \mathrm{~K}$ in effective temperature, $0.07 \mathrm{dex}$ in $\log (g)$ and 0.03 dex in $[\mathrm{Fe} / \mathrm{H}]$. Figure 3 shows the histogram of the $\mathrm{Mg}$ and $\mathrm{Y}$ abundances dispersion for the repeated spectra. The third quantile of the distribution is at $0.06 \mathrm{dex}$ for $\mathrm{Mg}$ and $0.10 \mathrm{dex}$ for Y.

Finally, external errors have been evaluated by comparing our abundance estimations to literature samples having a statistically relevant overlap with our data set. Figure 4 shows the histogram of the difference between our $\mathrm{Mg}$ abundance estimations and the Mikolaitis et al. (2014) values. The standard deviation of the difference is 0.08 dex. We note that Mikolaitis et al. (2014) use the same AMBRE parameters as this work, but only the highest $\mathrm{S} / \mathrm{N}$ spectra per star has been considered, contrary to our approach. In addition, they use a different abundance estimation method, and a different reference spectra grid. Figure 5 presents the histogram of the differences between our Y abundance estimations and the Delgado Mena et al. (2017) values (935 stars in common). In this case, the atmospheric parameters, the abundance estimation method and the reference theoretical spectra used by Delgado Mena et al. (2017) differ from those used in this work. The standard deviation of the $\mathrm{Y}$ abundance difference is 0.12 dex. 

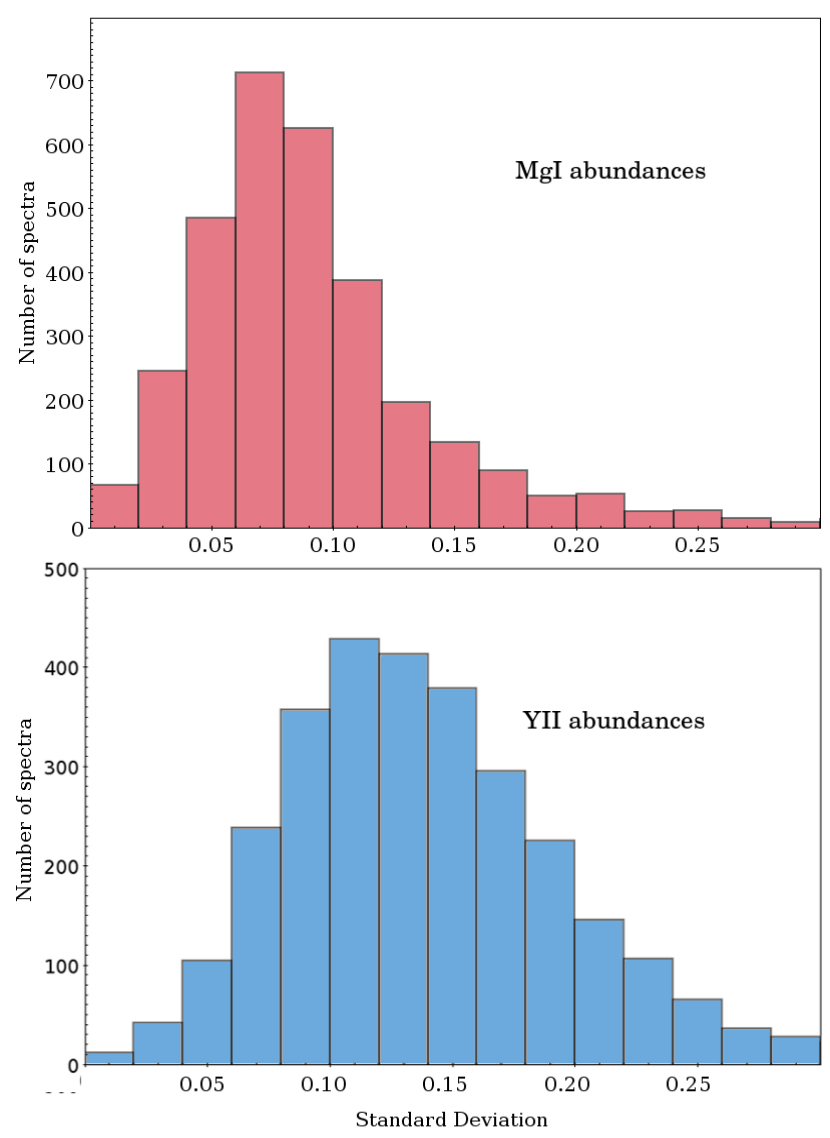

Fig. 2. Distribution of standard deviation for the estimation of $[\mathrm{Y} / \mathrm{H}]$ and $[\mathrm{Mg} / \mathrm{Fe}]$ from different lines. Top panel: distribution of the standard deviation of $[\mathrm{Mg} / \mathrm{Fe}]$ for each spectrum, calculated as median value by five spectral lines from Table 1. Bottom panel: as above, but for $[\mathrm{Y} / \mathrm{H}]$ abundances, calculated from eight spectral lines.

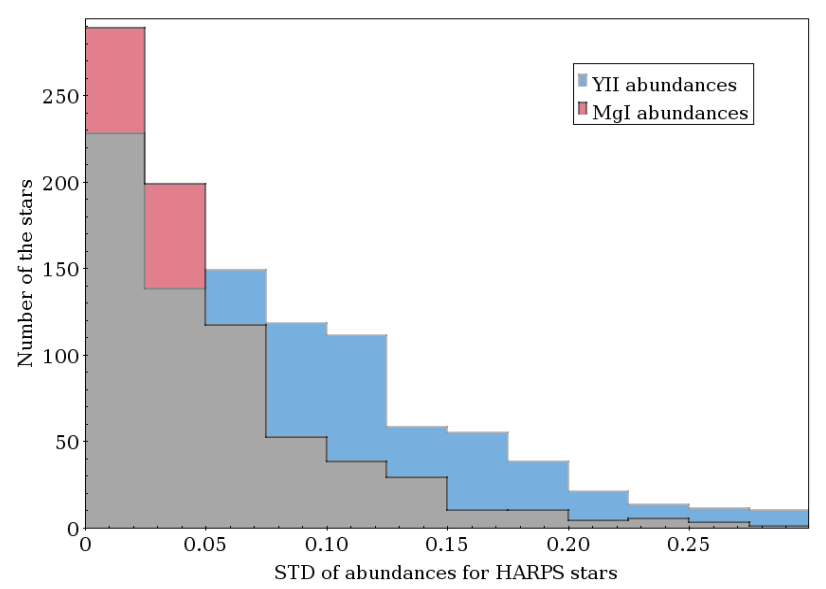

Fig. 3. Distribution of standard deviation of $[\mathrm{Y} / \mathrm{H}]$ and $[\mathrm{Mg} / \mathrm{Fe}]$ abundances for HARPS stars repeated observations: MgI (red), YII (blue), area in common (grey).

\section{Age dependence of the $[\mathrm{Y} / \mathrm{Mg}]$ abundance}

In this section, we analyse the time dependence of the $[\mathrm{Y} / \mathrm{Mg}]$ abundance as a function of metallicity, age, and disc component membership (thin or thick disc). The stars have been tagged as belonging to the thin disc, the thick disc, or the high-alpha metal-rich population following the chemical classification of Mikolaitis et al. (2017), which is based on its $[\mathrm{Mg} / \mathrm{Fe}]$ chemical index.

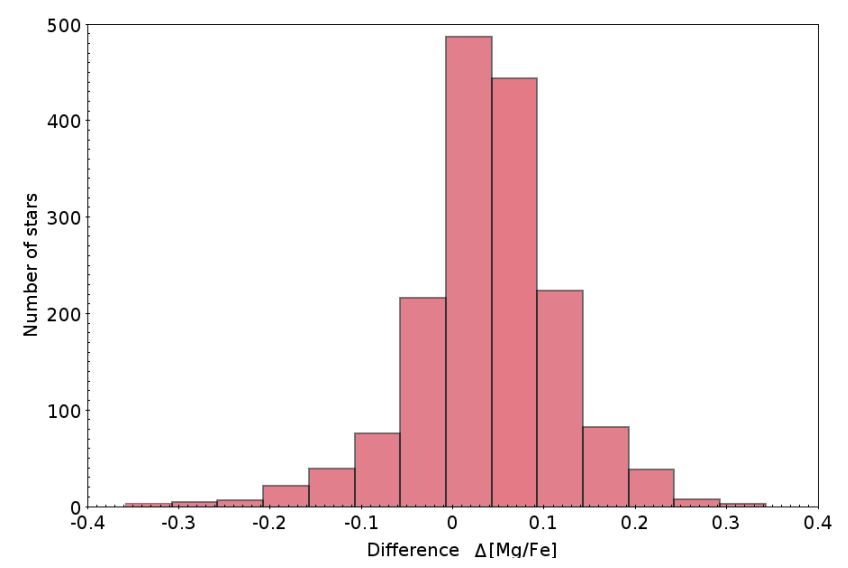

Fig. 4. Distribution of the difference in $[\mathrm{Mg} / \mathrm{Fe}]$ abundances between our AMBRE/HARPS results and those of Mikolaitis et al. (2017).

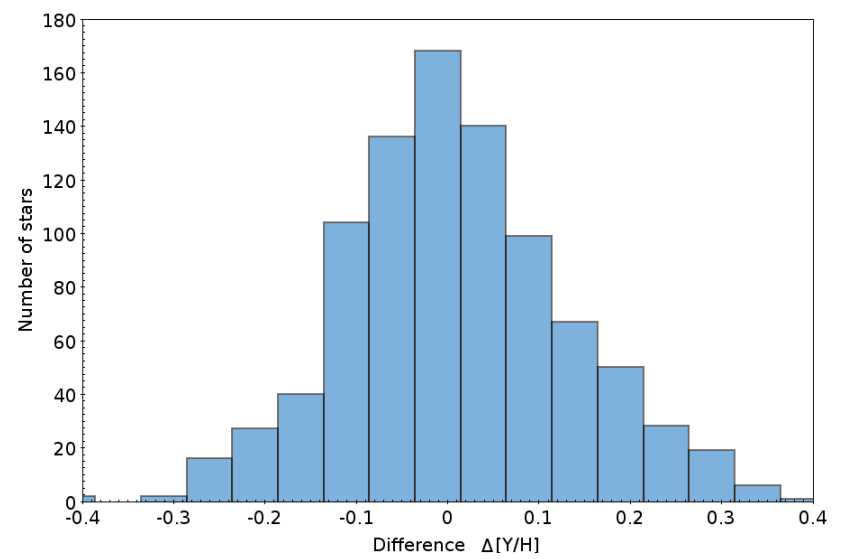

Fig. 5. Distribution of the difference between $[\mathrm{Y} / \mathrm{H}]$ abundances between AMBRE/HARPS and the results from Delgado Mena et al. (2017).

First, Fig. 6 presents the abundances of $[\mathrm{Fe} / \mathrm{H}],[\mathrm{Y} / \mathrm{H}]$, and $[\mathrm{Mg} / \mathrm{Fe}]$ as a function of the stellar age for turn-off stars with errors lower than 0.1 dex in abundances. A linear fit for each abundance ratio is presented. Although the $[\mathrm{Fe} / \mathrm{H}]$ (upper panel) and the $[\mathrm{Y} / \mathrm{H}]$ abundances (middle panel) present clear negative slopes, both of them present an important dispersion with stellar age $(0.23$ and 0.21 , respectively). The $[\mathrm{Mg} / \mathrm{Fe}]$ abundance ratio (lower panel) presents a better correlation, with a dispersion of 0.054 , that makes the $[\mathrm{Mg} / \mathrm{Fe}]$ ratio a rather good proxy of the age. However, two different regimes seem to exist: stars younger than and older than $\sim 10$ Gyr (top panel of Fig. 6). As shown in previous studies (Haywood et al. 2013, Hayden et al. 2018), the trend of the $[\mathrm{Mg} / \mathrm{Fe}]$ ratio with stellar age seems to be steeper for thick disc stars (black squares) than for thin disc stars (grey circles). Thus, the $[\mathrm{Mg} / \mathrm{Fe}]$ abundance cannot be chosen as a robust age indicator for the thin disc population.

Second, Fig. 7 shows the $[\mathrm{Y} / \mathrm{Mg}]$ abundance ratio as a function of stellar age for three different subsamples of turn-off stars, progressively increasing the abundance error constraints. The upper panel presents the stars with errors under the level of $0.10 \mathrm{dex}$ for $[\mathrm{Mg} / \mathrm{Fe}]$ and $0.20 \mathrm{dex}$ for $[\mathrm{Y} / \mathrm{H}]$ (estimated from the standard deviation of multiple lines). The linear fit corresponds to the thin disc population with ages younger than $10 \mathrm{Gyr}$. At this step, the 10 Gyr value is only based on the approximative position separating thin and thick disc stars after the cleaning with the abundance errors. We recall that our thin/thick disc is the one 

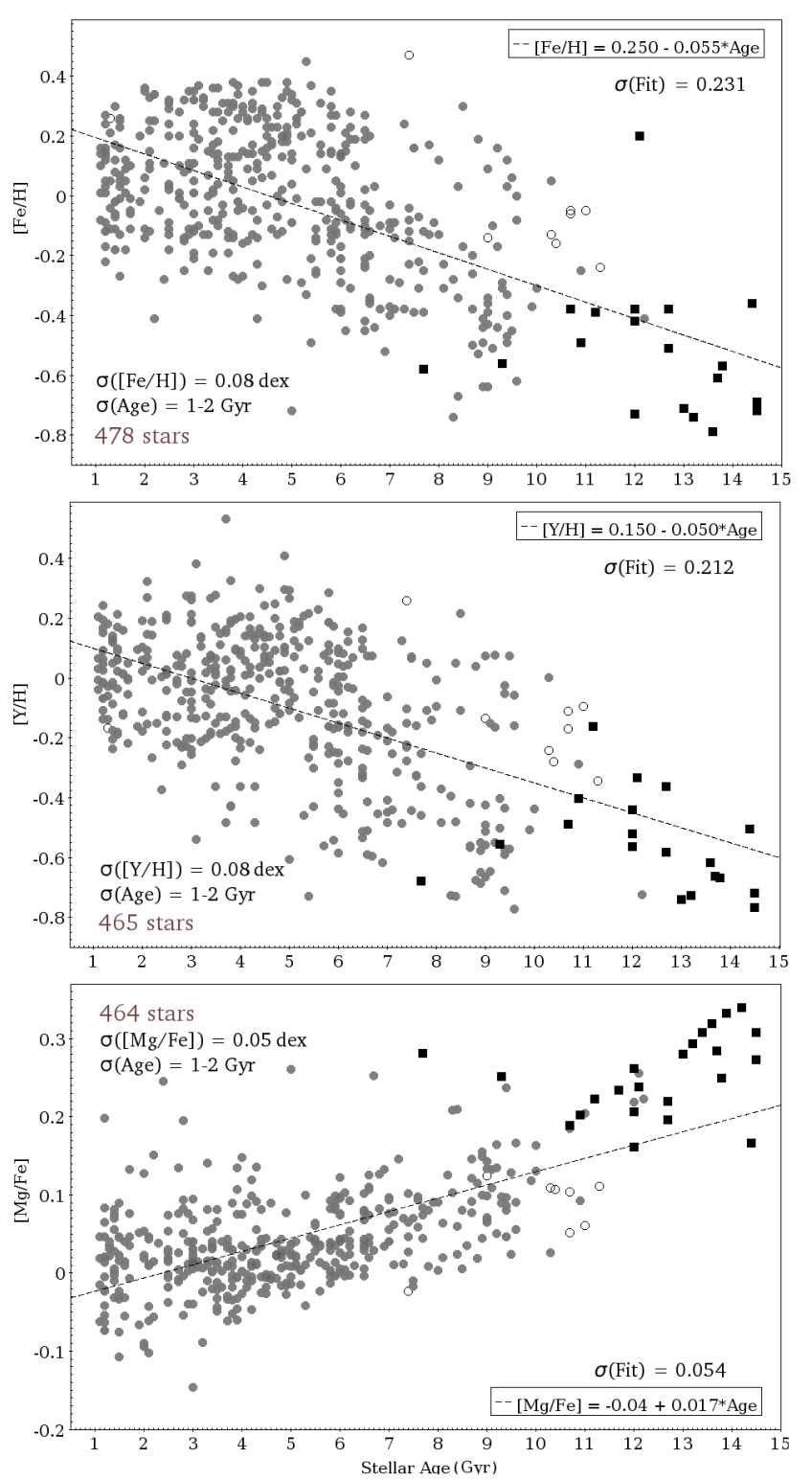

Fig. 6. Trends of the $[\mathrm{Fe} / \mathrm{H}],[\mathrm{Y} / \mathrm{H}]$, and $[\mathrm{Mg} / \mathrm{Fe}]$ abundances with age for the complete turn-off sample of AMBRE/HARPS data. Number of stars, coefficients of the fit, and total dispersion of the fit are given in each panel. Thin disc stars are plotted as filled circles, and thick disc stars as filled squares. Opened circles are high-alpha metal-rich objects, following the chemical classification of Mikolaitis et al. (2017).

presented in a trend with age is already clear. With respect to the thin disc population (circles), the thick disc stars (squares) older than 10-11 Gyr seem to be shifted to the thin disc population, although they present an anti-correlation $(-0.58 \mathrm{dex})$ with age. The thin disc versus thick disc differences are studied in greater detail in Sect. 7. The middle panel presents a sample with errors lower than 0.05 dex in $[\mathrm{Mg} / \mathrm{Fe}]$ and $0.10 \mathrm{dex}$ in $[\mathrm{Y} / \mathrm{H}]$. The correlation increases slightly to -0.62 dex due to smaller dispersion around the thin disc points. It is difficult to disentangle between the spread caused by the abundance and age errors and a possible natural spread due to metallicity because there is a metallicity dependence of the errors. The lower panel of Fig. 7, where the error selection is more severe, contains only stars in the metallicity interval $-0.4 \operatorname{dex}<[\mathrm{M} / \mathrm{H}]<0$ dex.

No clear trend with metallicity seems to be present, contrarily to the study of Feltzing et al. (2017). This difference could
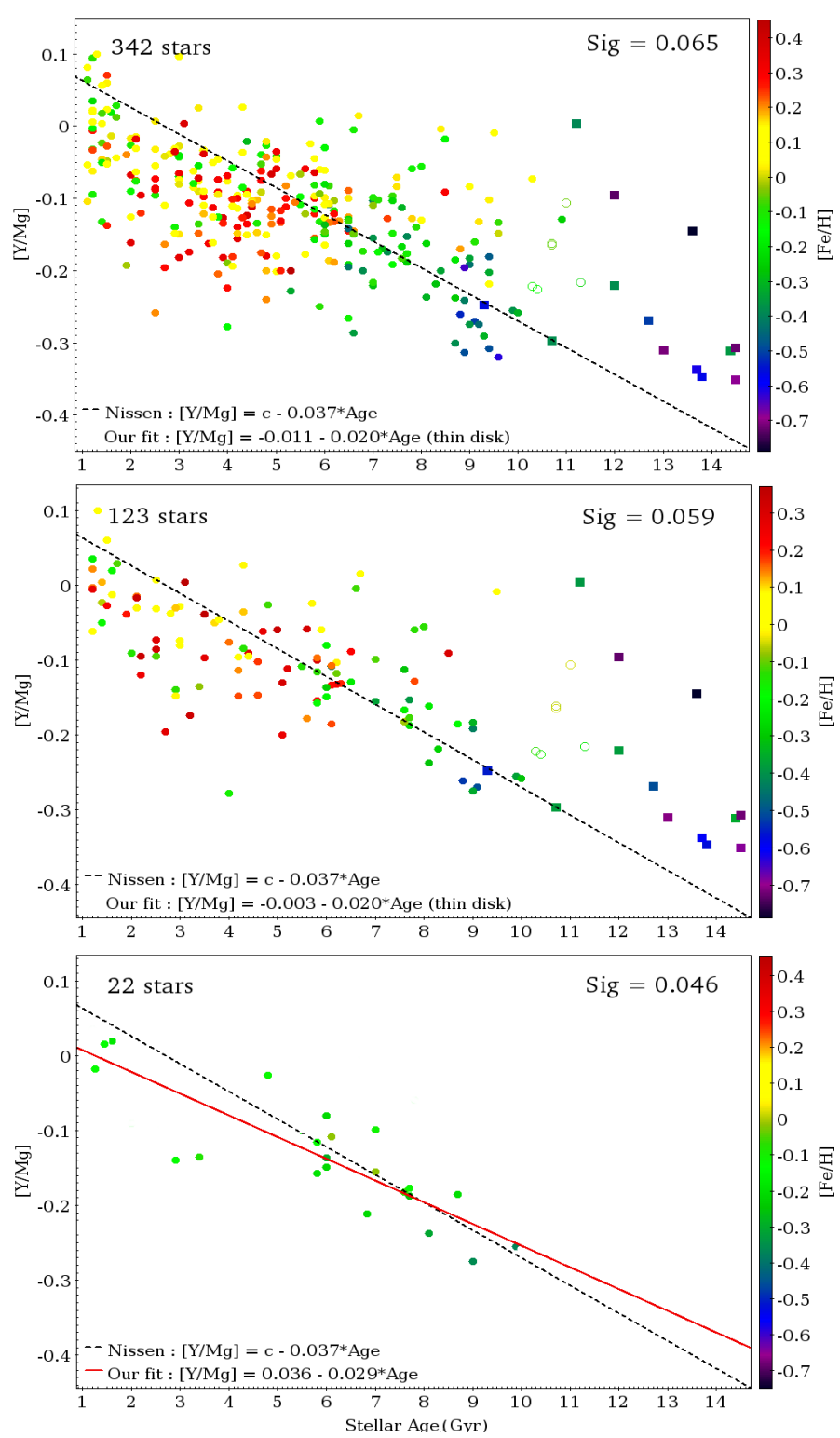

Fig. 7. $[\mathrm{Y} / \mathrm{Mg}]$ vs. stellar age dependence. Top panel: 342 stars with $\sigma_{\mathrm{YII}}<0.2$ and $\sigma_{\mathrm{MgI}}<0.1 \mathrm{dex} ;$ middle panel: 123 turn-off stars with $\sigma_{\mathrm{YII}}<0.1$ dex and $\sigma_{\mathrm{MgI}}<0.05$ dex; bottom panel: 22 turn-off thin disc stars with $\sigma_{\mathrm{YII}}<0.05$ dex and $\sigma_{\mathrm{MgI}}<0.05$ dex. The symbols are as in Fig. 6, and are also colour-coded for the stellar metallicity. The dotted line shows the (Nissen 2015) fit for solar type stars (here $c=0.1 \mathrm{dex}$ ); the red solid line (bottom panel) is the fit for the high-precision sample of thin disc stars.

be caused, first, by the spread in the data from Feltzing et al. (2017), which is higher than that used in our selection and can come from the fact that they are using a heterogeneous spectra data set (see Table 1 in Bensby et al. 2014) and/or the lower spectroscopic resolution of the majority of their data. Second, we have seen that the errors in the abundances are temperature and metallicity dependent, which makes it difficult to compare the two data sets in detail. Also, for metallicities lower than around $-0.5 \mathrm{dex}$, the thin and the thick disc regimes overlap, making the interpretation more complex, especially if the two populations have different slopes of the [Y/Mg] abundance versus age.

Finally, we point out that many of the young and intermediate age thin disc stars are metal-rich objects with very crowded spectra. Therefore, a final selection of a limited sample of stars 


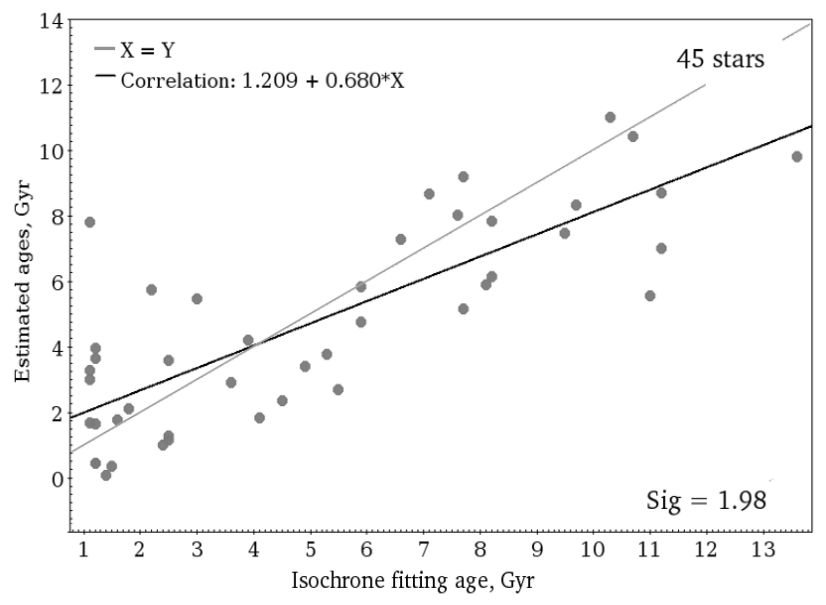

Fig. 8. Comparison of stellar ages derived from the $[\mathrm{Y} / \mathrm{Mg}]$ ratio with isochrone fitting ages for a sample of stars not used to infer the [Y/Mg]age relation. The thick line represents the final correlation; the thin grey line is a diagonal.

Table 3. Results of the fitting with stellar age for different elements.

\begin{tabular}{cccc}
\hline \hline fit & A & B * Age & $\sigma_{\text {fit }}$ \\
\hline$[\mathrm{Y} / \mathrm{Mg}]_{\text {thin }}$ & $0.036 \pm 0.005$ & $-0.029 \pm 0.002$ & 0.046 \\
{$[\mathrm{Y} / \mathrm{Mg}]_{\text {thick }}$} & $0.833 \pm 0.006$ & $-0.082 \pm 0.002$ & 0.076 \\
\hline
\end{tabular}

Notes. The coefficients for $\mathrm{Fe}, \mathrm{Y}$, and $\mathrm{Mg}$ abundances are calculated for all turn-off stars with all available ages, the fit of $[\mathrm{Y} / \mathrm{Mg}]$ is given for the sample of 325 turn-off stars from thin disc (Fig. 9).

hotter than $5700 \mathrm{~K}$ and having $[\mathrm{M} / \mathrm{H}]$ less than 0.0 dex was performed. This selection avoids problems of blending and continuum placement that could be affecting the spectral analysis. In addition, we only kept stars with errors lower than 0.05 dex both in the $[\mathrm{Mg} / \mathrm{Fe}]$ and in the $[\mathrm{Y} / \mathrm{H}]$ abundance. This high-precision sample is shown in the lower panel of Fig. 7. Clearly, a clean anti-correlation appears $(-0.82)$ down to 9 Gyr. The final fit of this correlation is

$$
[\mathrm{Y} / \mathrm{Mg}]_{\text {thin disc }}=(0.036 \pm 0.005)-(0.029 \pm 0.0015) \times \text { Age }
$$

This fit approaches that of Nissen (2015) and Tucci Maia et al. (2016) for solar twins within a slightly lower value of the slope. Reducing the parameter space covered by the data set helps to reduce the internal error spread, including internal biases from one type of star to another.

\section{Chemical clock: inverting the $[\mathrm{Y} / \mathrm{Mg}]-$ age relation.}

The clean [Y/Mg]-age relation derived in Sect. 5 can be used to estimate stellar ages for thin disc stars, replacing isochrone fitting outside the ranges of applicability (e.g. main sequence stars outside the turn off). The precision in the derived age can be estimated by propagating the errors in Eq. (1). As an example, for a star with $[\mathrm{Y} / \mathrm{Mg}]=(-0.1 \pm 0.05)$ dex the estimation of error in age is around $2 \mathrm{Gyr}$.

To test this chemical clock we have considered 45 AMBRE/HARPS main sequence and subgiant branch stars slightly outside the conditions of the turn-off definition proposed in Hayden et al. (2018). We derived isochrone fitting ages for these stars, which were not used in the analysis of Sect. 5,

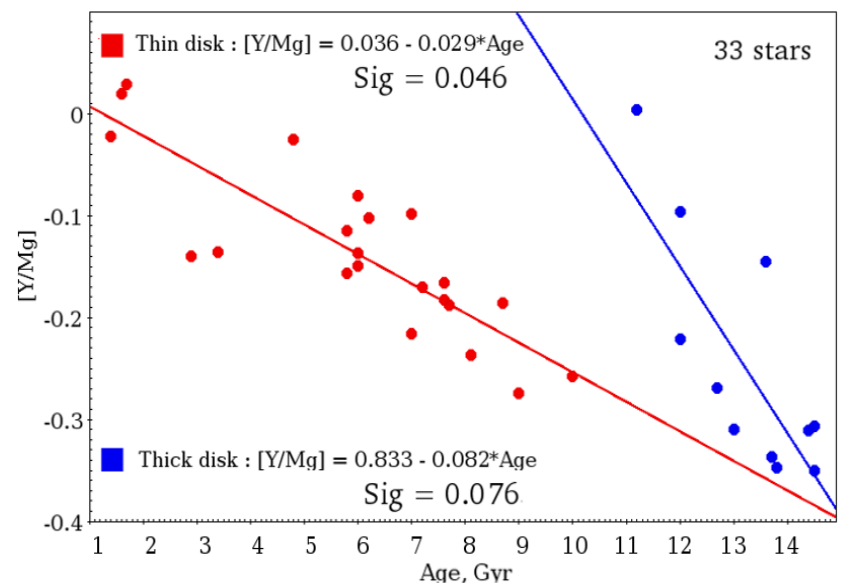

Fig. 9. $[\mathrm{Y} / \mathrm{Mg}]$ vs. age fit for thin and thick disc stars.

following the procedure of Hayden et al. (2018). In parallel [Y/Mg]-based ages were derived. Figure 8 shows the comparison of the two sets of ages. A good correlation appears, with a standard deviation of the difference equal to 2.4 Gyr. The zero point of the difference seems to be within the error for stars younger than $10 \mathrm{Gyr}$, like those of the thin disc used to derive the relation. In conclusion, the $[\mathrm{Y} / \mathrm{Mg}]$ abundance ratio can be used as an age proxy for the thin disc stars with reasonable precision, and it can replace more precise age estimations when they are not available.

\section{Differences between the thin and the thick disc.}

The dependence of $[\mathrm{Y} / \mathrm{Mg}]$ on stellar age is based on the chemical evolution of the analysed population, which means that different galactic components could present different trends with age depending, among other things, on their star formation history. In the previous sections, we focus the analysis on thin disc stars, which represent the majority of the AMBRE/HARPS turnoff data.

However, in Sect. 5 we point out the shift that thick disc stars seem to present with respect to the thin disc, causing a discontinuity in the $[\mathrm{Y} / \mathrm{Mg}]$ correlation with age. Figure 8 shows the high-precision thin disc data set together with the thick disc set, but relaxing the errors to $0.1 \mathrm{dex}$ in $[\mathrm{Mg} / \mathrm{Fe}]$ and $0.20 \mathrm{dex}$ in $[\mathrm{Y} / \mathrm{H}]$. This allows us to include more metal-poor stars in the sample. A similar difference between thick and thin disc appears in the evolution of the $[\mathrm{Mg} / \mathrm{Fe}]$ ratio with time in the studies of Haywood et al. (2013) and Hayden et al. (2018), highlighting differences in the chemical evolution of these two populations, with the thick disc probably presenting a higher star formation rate. On the other hand, very few literature reports exist regarding the [Y/Mg] trend with age, Delgado Mena et al. (2018) find a similar step in the $[\mathrm{Y} / \mathrm{Mg}]$ for stars with an age older than $10 \mathrm{Gyr}$.

The final derived trends for the thin and thick disc populations are summarised in Table 3.

\section{Summary and conclusions}

The analysis presented in this paper confirms the sensitivity of the $[\mathrm{Y} / \mathrm{Mg}]$ abundance ratio to stellar age. Thanks to a sample with a high precision of chemical abundances and isochrone ages, the correlation of the $[\mathrm{Y} / \mathrm{Mg}]$ abundance with age has been evaluated for thin disc and thick disc stars in the solar neighbourhood. 
First, we have shown the importance of reducing the relative errors in the abundances by selecting i) stellar types that are reliable to analyse (e.g. less crowded spectra, easier continuum estimation) and ii) reduced parameter space of the studied samples to minimise internal biases.

Second, a clear correlation between $[\mathrm{Y} / \mathrm{Mg}]$ and age has been found, with a trend similar to those found in previous studies for solar-type stars (Nissen 2015; Tucci Maia et al. 2016). For the thin disc stars, no particular trend with metallicity seems to exist. We have tested the obtained ratio for stellar age dating through the use of the $[\mathrm{Y} / \mathrm{Mg}]$ chemical diagnostic, and a precision of around $2 \mathrm{Gyr}$, in comparison with existing data, was achieved.

Finally, for the first time, we have detected a difference between the thin disc and the thick disc population in the [Y/Mg] dependence with age. As expected from the different chemical evolution history for these two populations, different trends of the $[\mathrm{Y} / \mathrm{Mg}]$ abundance with age seem to exist. A threshold around $10 \mathrm{Gyr}$ appears in our sample, revealing a chemical discontinuity between the thick and the thin disc. The population of thick disc stars seems to have a steeper trend with age. To clarify this, more investigations on the thick disc population are needed.

We conclude that in the era of large surveys of the Milky Way for which stellar age estimations are a crucial ingredient, chemical diagnostics like the one presented here open new paths for stellar dating.

Acknowledgements. The spectra calculations were performed with the highperformance computing facility MESOCENTRE, hosted by OCA. This work has made use of data from the European Space Agency (ESA) mission Gaia (https://www.cosmos.esa.int/gaia), processed by the Gaia Data Processing and Analysis Consortium (DPAC; https://www.cosmos.esa.int/ web/gaia/dpac/consortium). We acknowledge financial support form the ANR 14-CE33-014-01. Funding for the DPAC has been provided by national institutions, in particular the institutions participating in the Gaia Multilateral Agreement.

\section{References}

Adibekyan, V. Z., Sousa, S. G., Santos, N. C., et al. 2012, A\&A, 545, A32 Alonso, A., Arribas, S., \& Martinez-Roger, C. 1996, A\&A, 313, 873 Anders, F., Chiappini, C., Santiago, B. X., et al. 2018, A\&A, 619, A125 Bailer-Jones, C. A. L., Andrae, R., Arcay, B., et al. 2013, A\&A, 559, A74 Bensby, T., Feltzing, S., \& Oey, M. S. 2014, A\&A, 562, A71
Bijaoui, A. 2012, in Seventh Conference on Astronomical Data Analysis, eds. J.-L. Starck, \& C. Surace, 2

Bland-Hawthorn, J., \& Freeman, K. 2014, The Origin of the Galaxy and Local Group, Saas-Fee Advanced Course (Berlin Heidelberg: Springer-Verlag), 37,1

Chabrier, G. 2003, PASP, 115, 763

da Silva, R., de Porto Mello, G. F., \& Milone, A. C., et al. 2012, A\&A, 542, A84 de Laverny, P., Recio-Blanco, A., Worley, C. C., \& Plez, B. 2012, A\&A, 544, A126

de Laverny, P., Recio-Blanco, A., Worley, C. C., et al. 2013, The Messenger, 153, 18

Delgado Mena, E., Tsantaki, M., Adibekyan, V. Z., et al. 2017, A\&A, 606, A94 Delgado Mena, E., Tsantaki, M., Zh. Adibekyan, V., et al. 2018, in IAU Symp., eds. A. Recio-Blanco, P. de Laverny, A. G. A. Brown, et al., 330, 156

De Pascale, M., Worley, C. C., de Laverny, P., et al. 2014, A\&A, 570, A68

Dotter, A., Chaboyer, B., Jevremović, D., et al. 2008, ApJS, 178, 89

Feltzing, S., Howes, L. M., McMillan, P. J., \& Stonkutè, E. 2017, MNRAS, 465, L109

Gaia Collaboration (Brown, A. G. A., et al.) 2016a, A\&A, 595, A2

Gaia Collaboration (Prusti, T., et al.) 2016b, A\&A, 595, A1

González Hernández, J. I., \& Bonifacio, P. 2009, A\&A, 497, 497

Guiglion, G., de Laverny, P., Recio-Blanco, A., et al. 2016, A\&A, 595, A18

Guiglion, G., de Laverny, P., Recio-Blanco, A., \& Prantzos, N. 2018, A\&A, 619, A143

Gustafsson, B., Edvardsson, B., Eriksson, K., et al. 2008, A\&A, 486, 951

Guzik, J. A., Kaye, A. B., Bradley, P. A., Cox, A. N., \& Neuforge, C. 2000, ApJ, 542, L57

Hannaford, P., Lowe, R. M., Grevesse, N., Biemont, E., \& Whaling, W. 1982, ApJ, 261, 736

Hayden, M. R., Recio-Blanco, A., de Laverny, P., et al. 2018, A\&A, 609, A79

Haywood, M., Di Matteo, P., Lehnert, M. D., Katz, D., \& Gómez, A. 2013, A\&A, 560, A109

Hoyle, F., \& Fowler, W. A. 1960, ApJ, 132, 565

Jørgensen, B. R., \& Lindegren, L. 2005, A\&A, 436, 127

Luri, X., Torra, J., \& Figueras, F. 1992, A\&A, 259, 382

McWilliam, A., Wallerstein, G., \& Mottini, M. 2013, ApJ, 778, 149

Mikolaitis, Š., Hill, V., Recio-Blanco, A., et al. 2014, A\&A, 572, A33

Mikolaitis, Š., de Laverny, P., Recio-Blanco, A., et al. 2017, A\&A, 600, A22

Nissen, P. E. 2015, A\&A, 579, A52

Perryman, M., Hartman, J., Bakos, G. Á., \& Lindegren, L. 2014, ApJ, 797, 14

Pesnell, W. D. 1987, ApJ, 314, 598

Recio-Blanco, A., Bijaoui, A., \& de Laverny, P. 2006, MNRAS, 370, 141

Recio-Blanco, A., de Laverny, P., Kordopatis, G., et al. 2014, A\&A, 567, A5

Recio-Blanco, A., de Laverny, P., Allende Prieto, C., et al. 2016, A\&A, 585, A93

Robin, A. C., Luri, X., Reylé, C., et al. 2012, A\&A, 543, A100

Schaltenbrand, R. A. 1974, A\&AS, 18, 27

Slumstrup, D., Grundahl, F., Brogaard, K., et al. 2017, A\&A, 604, L8

Spina, L., Meléndez, J., Karakas, A. I., et al. 2016, A\&A, 593, A125

Tucci Maia, M., Ramírez, I., Meléndez, J., et al. 2016, A\&A, 590, A32

Valls-Gabaud, D. 2014, EAS Pub. Ser., 65, 225 